HIV IN CHINA

\title{
Attitudes to HIV and HIV testing in high prevalence areas of China: informing the introduction of voluntary counselling and testing programmes
}

\author{
T Hesketh, L Duo, H Li, A M Tomkins
}

Sex Transm Infect 2005;81:108-112. doi: 10.1136/sti.2004.009704

See end of article for authors' affiliations

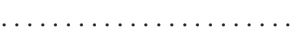

Correspondence to: Therese Hesketh, Centre for International Child Health, Institute of Child Health, 30 Guilford Street, London WCIN 1EH; UK; t.hesketh@ich.ucl.ac.uk

Accepted for publication 5 July 2004
Objectives: To assess knowledge and attitudes towards HIV and its testing among pregnant women and health professionals in Yunnan Province, south west China, to inform the introduction of voluntary counselling and testing (VCT) programmes.

Methods: The study design was a cross sectional survey using self completion questionnaires. It was carried out in 12 hospitals in four high prevalence areas of Yunnan Province. Questionnaires were completed under examination conditions by health professionals, and at the routine antenatal examination by pregnant women.

Results: Completed questionnaires were obtained from 840 pregnant women and 780 health professionals. Knowledge of HIV and its modes of transmission were good in health professionals but patchy in pregnant women. The weakest area in both groups was knowledge of maternal to child transmission. There was strong support for compulsory testing in pregnancy and at the premarital examination. But attitudes towards HIV/AIDS were negative: $23 \%$ of health professionals and $45 \%$ of pregnant women thought HIV was a disease of "low class and illegal" people, $48 \%$ of health professionals and $59 \%$ of pregnant women thought that HIV positive individuals should not be allowed to get married, and $30 \%$ of the health professionals were not willing to treat an HIV positive individual. Levels of knowledge were higher and attitudes more positive in younger health professionals and better educated pregnant women.

Conclusions: Community education programmes and intensive training of health workers must precede or accompany VCT programmes. They must particularly address negative attitudes towards people with HIV. Pilot VCT programmes are now under way in two of the areas studied.
O fficial estimates put the number of people living with HIV/AIDS (PLWHA) in China at over one million, rising at a rate of over $30 \%$ per year. UNAIDS predicts that there could be 10 million to 15 million PLWHA in China by $2010 .{ }^{1}$ This could make China the major contributor to the global HIV epidemic. ${ }^{2}$ Urgent prevention and control measures are clearly needed and the Chinese government has now openly acknowledged this. Among the measures under discussion is the introduction of programmes of voluntary counselling and testing (VCT). In China appropriate and acceptable programmes are especially important, because at present testing is being carried out on a very limited basis. Most of the 40650 individuals who had been identified as HIV positive by the end of 2002 were tested as part of the Ministry of Health Sentinel Surveillance system which targets high risk groups, including injecting drug users, commercial sex workers, and STI clinic attendees. ${ }^{3}$ It is carried out on a compulsory anonymous unlinked basis, though it is known that anonymity is not always maintained and feedback does sometimes take place. ${ }^{4}$

Evidence from research in both developed and developing countries shows that VCT leads to earlier access to HIV therapy, which in turn increases its effectiveness and that VCT reduces risk behaviour. ${ }^{5}$ In many countries VCT programmes are playing an increasing part in prevention and care strategies. ${ }^{5-7}$ With locally produced, cheaper antiretrovirals now more widely available in China, there is a clear need for a systematic approaches to testing, which are based on VCT models, and which include low risk populations. But little is known about the acceptability and likely uptake of VCT in China or the best vehicles for testing. Opportunistic testing is one approach, and VCT is recommended as part of routine antenatal care in many countries. ${ }^{8}$ In China there is also the opportunity for testing at the premarital examination. This health examination, which was still compulsory in most of China at the time of this study, focuses on inheritable and reproductive disorders, including sexually transmitted infection. ${ }^{9}$ One advantage is the access to men, especially useful in China where the reported male:female ratio for HIV is $4: 1 .^{3}$

There are a number of prerequisites for successful VCT programmes. Apart from the need for clear protocols for counselling, testing, and treatment there is also the need for: (a) awareness and knowledge of HIV in the community, (b) acceptability of VCT to potential recipients and to the health professionals who must administer it, and (c) willingness of health professionals to treat HIV positive individuals. ${ }^{6} 710$ This study was carried out to explore these three questions among pregnant women and health professionals in areas where VCT was planned as part of routine antenatal care or at the premarital examination. It was carried out in collaboration with the maternal and child health department of Yunnan Bureau of Public Health.

\section{METHODS}

The study was carried out in four areas of Yunnan Province, which is located in south west China, bordering Burma, Laos, and Vietnam, and close to the "Golden Triangle." It has the highest reported prevalence of HIV in China, accounting for

Abbreviations: PLWHA, people living with HIV/AIDS; VCT, voluntary counselling and testing 
$40 \%$ of all official reports, with around $70 \%$ of these in injecting drug users. ${ }^{3}$ The four areas comprised a district in Kunming, the provincial capital, a district in Dali in central Yunnan, a county in Xishuangbanna prefecture close to the Laotian border, and a county in Derhong prefecture on the Burmese border. Except for the district in Kunming all are known as areas where drugs and commercial sex are common. There are limited population based data for HIV in these areas, but all are regarded as areas of high HIV prevalence by Chinese standards. A prevalence of $0.8 \%$ has been reported in premarital examination attendees in Dali and $0.7 \%$ in Kunming. ${ }^{11}$ A small study in Derhong prefecture found rates of $1.4 \%$ in antenatal clinic in Kunming. ${ }^{12}$ The aim was to obtain a sample size of 200 pregnant women and 200 health professionals in each area. To achieve this in each area three hospitals, which provided antenatal care and/or premarital examination services, were asked to participate and all agreed.

Questionnaires were developed by the research team with questions divided into three subject areas: knowledge, attitudes to testing, and attitudes to PLWHA. Questions on knowledge were drawn from existing tools ${ }^{13}$ and were included on the basis that knowledge is essential to informed choices about testing. Questions on attitudes were developed specifically for the study and included personal reactions to HIV testing, willingness to disclose status, and reactions to a positive test. Attitudes to PLWHA included specific questions to health professionals about willingness to treat HIV patients. The aim was to make the questionnaire for health professionals and pregnant women as similar as possible for comparison purposes. The draft questionnaire was piloted among 40 health professional and pregnant women and modifications were made accordingly.

Health professionals completed the questionnaire anonymously under examination conditions. After completion a seminar was held by the researchers (DL or LH) to discuss issues raised by the questionnaire.

The questionnaire for pregnant women was administered by research assistants during routine antenatal examinations at the project sites. All women attending during the study period (April-May 2002) were asked to complete the questionnaire. They gave verbal consent and it was explained that non-compliance would not in any way influence their clinical management. They were given the explicit option to refuse though none did. Respondents were encouraged to complete the questionnaire themselves but researchers assisted where necessary. For respondents illiterate in Chinese research assistants with knowledge of local languages administered the questionnaire verbally and transcribed the responses. This was necessary for 218 women or $25 \%$. Ethical approval for the study was obtained from the
Yunnan Bureau of Public Health and the ethics committee of the Institute of Child Health, University College London.

\section{Analysis}

For analysis purposes age was adjusted as a dichotomous variable $>30$ years and $\geqslant 30$ years, and education to completion or non-completion if middle school. Knowledge questions were analysed separately and by percentage score for the number of correct answers. This was adjusted as a continuous variable. Unadjusted odds ratios (Pearson $\chi^{2}$ and $95 \%$ confidence intervals) were calculated and then multivariate analysis was used to adjust where appropriate for age, education, sex, and area.

\section{RESULTS}

Characteristics of the study population (table 1)

There were completed questionnaires from 840 pregnant women and 780 health professionals, a 98\% response rate based on the completion of selected key variables. The sociodemographic composition is given in table 1. Almost one third of the respondents were from ethnic minorities (mostly Dai, Bai, Hui, and Yi) reflecting the ethnic diversity in Yunnan Province. Sixty seven per cent of the health professionals were nurses, explaining the female preponderance among the study subjects.

\section{HIV/AIDS knowledge (table 2)}

Health professionals' knowledge was good overall. The mean score on the knowledge test was $80 \%$ (range $35-100 \%$ ). But there was some misinformation: kissing was thought to be a transmission route by $24 \%$, mosquitoes by $22 \%$. The weakest area of knowledge was in the areas of maternal-child transmission: only 57\% were aware that HIV was transmitted in breast milk, only $22 \%$ that maternal to child transmission can be reduced by drugs and only 19\% that caesarean section is the preferred mode of delivery for HIV positive women (caesarean section is easily accessible in all these areas). There were no significant differences in knowledge scores by sex $(p=0.09)$ or area $(p=0.2)$ but younger age (OR 1.5, CI: 1.3 to 1.7) was an independent predictor of a higher knowledge score.

The knowledge of the pregnant women was patchy with a high proportion of "don't knows." Their mean score was $57 \%$ (range $12 \%-85 \%$ ). Over $70 \%$ correctly identified the major modes of transmission and $62 \%$ were aware of the protective effects of condoms, but only $15 \%$ knew of the risks of breast feeding. Knowledge scores were consistently higher in mothers in Derhong (OR 1.8, CI: 1.5 to 2.0) compared with the three other areas grouped together. Here formal education levels are lowest, but there have been recent intensive HIV/AIDS education campaigns. Outside Derhong higher

\begin{tabular}{|c|c|c|c|}
\hline & & \multirow{2}{*}{$\begin{array}{l}\text { Health professionals } \\
\text { No }(\%)\end{array}$} & \multirow{2}{*}{$\begin{array}{l}\text { Pregnant women } \\
\text { No }(\%)\end{array}$} \\
\hline & & & \\
\hline \multirow[t]{4}{*}{ Area } & Kunming & $238(30)$ & $227(27)$ \\
\hline & Dali & $201(26)$ & $203(24)$ \\
\hline & Yingjiang & $215(27)$ & $221(26)$ \\
\hline & Xishuangbanna & $126(17)$ & $183(22)$ \\
\hline \multirow[t]{2}{*}{ Age } & Mean (years) (SD) & $32(8.5)$ & $25.6(3.7)$ \\
\hline & Range & $17-65$ & $17-42$ \\
\hline \multirow[t]{2}{*}{ Sex } & Male & $128(21.3)$ & \\
\hline & Female & $656(78.7)$ & \\
\hline \multirow[t]{2}{*}{ Education } & Completed middle school & $632(81)$ & $294(35)$ \\
\hline & Not completed middle school & $148(19)$ & $546(65)$ \\
\hline \multirow[t]{2}{*}{ Ethnicity } & Han & $538(69)$ & $571(68)$ \\
\hline & Others: Bai, Dai, Hui, Yi & $242(31)$ & $269(32)$ \\
\hline
\end{tabular}


Table 2 Knowledge questions about HIV: responses by percentage

\begin{tabular}{|c|c|c|c|c|}
\hline & \multicolumn{2}{|c|}{ Health professionals } & \multicolumn{2}{|c|}{ Pregnant women } \\
\hline & Yes & No & Yes & No \\
\hline Have you heard of an infection called HIV? & 100 & 0 & 100 & 0 \\
\hline Do you think that HIV causes a fatal disease? & 100 & 0 & 100 & 0 \\
\hline \multicolumn{5}{|l|}{ You can be infected with HIV } \\
\hline From shaking hands & 1 & 97 & 8 & 61 \\
\hline Using toilets & 12 & 80 & 20 & 52 \\
\hline Sharing food with HIV positive people & 6 & 88 & 9 & 73 \\
\hline Chopsticks & 10 & 85 & 11 & 56 \\
\hline Kissing & 24 & 66 & 50 & 30 \\
\hline Sexual intercourse & 98 & 1 & 74 & 2 \\
\hline Sharing unsterilised needles & 96 & 3 & 74 & 8 \\
\hline Blood transfusion & 96 & 3 & 75 & 7 \\
\hline From a mosquito bite & 22 & 73 & 44 & 27 \\
\hline From mother to baby across the placenta & 98 & 2 & 80 & 6 \\
\hline From mother to baby through breast feeding & 57 & 37 & 35 & 49 \\
\hline \multicolumn{5}{|l|}{ Do you agree with the following statements? } \\
\hline HIV infected individuals can be determined by their appearance & 6 & 90 & 15 & 44 \\
\hline $\begin{array}{l}\text { The correct use of condoms can protect against sexual transmission } \\
\text { of HIV. }\end{array}$ & 93 & 4 & 62 & 11 \\
\hline If one family member has HIV the others will become infected & 9 & 85 & 23 & 55 \\
\hline Homosexual men are at higher risk of acquiring HIV & 80 & 18 & 55 & 5 \\
\hline $\begin{array}{l}\text { Is it true that HIV can only spread among low class and "illegal" } \\
\text { people }\end{array}$ & 2 & 95 & 13 & 68 \\
\hline HIV/AIDS is now curable & 25 & 64 & 20 & 38 \\
\hline $\begin{array}{l}\text { There is now a medicine which can reduce the risk of maternal to } \\
\text { child transmission }\end{array}$ & 22 & 55 & NA & NA \\
\hline $\begin{array}{l}\text { Vaginal delivery is recommended for HIV positive women. } \\
\text { Caesarean section should be avoided }\end{array}$ & 19 & 59 & NA & NA \\
\hline
\end{tabular}

education level was the only predictor of better knowledge (ORl.6, CI: 1.3 to 1.8 ).

\section{Attitudes to PLWHA (table 3)}

Attitudes to PLWHA indicate considerable intolerance even among the health professionals: $23 \%$ of health professionals and $73 \%$ of pregnant women believed that HIV/AIDS is a condition of "low class and illegal people," the majority of both groups would keep their distance from an HIV positive acquaintance $(60 \%$ and $75 \%)$ respectively, $29 \%$ of health professionals and 59\% of pregnant women think individuals with HIV should not be allowed to get married, with $43 \%$ and $66 \%$ respectively saying that they should not be allowed to have children. In comparison $22 \%$ and $13 \%$ respectively thought that people with other inheritable disorders should not have children either.

More tolerant responses were consistently associated with being younger in health professionals, with better education in pregnant women, and being an inhabitant of Derhong in both groups. But there was no relation to level of knowledge as defined by knowledge score.

The negative attitudes were also evident in professional approaches: $30 \%$ were not willing to treat people with HIV/ AIDS, and $81 \%$ would prefer not to, though younger ones were much more willing (OR 2.0, CI 1.8 to 2.3) as were those from Derhong (OR 1.8, CI: 1.5 to 2.0).

\section{Attitudes to testing (table 4)}

Testing for HIV was supported by the overwhelming majority of the participants, both health professionals and pregnant women. Testing at the premarital examination was favoured over testing in the antenatal period in both groups. But perhaps surprisingly, compulsory testing was favoured over voluntary testing in both groups and in both settings (antenatal clinic and at the premarital examination). The overwhelming majority of health professionals (91\%) and pregnant women $(77 \%)$ claimed to be willing to undergo testing for HIV. A slightly smaller proportion would tell their

Table 3 Attitudes to PLWHA: responses by percentage

\begin{tabular}{|c|c|c|c|c|}
\hline & \multicolumn{2}{|c|}{ Health professionals } & \multicolumn{2}{|c|}{ Pregnant women } \\
\hline & Yes & No & Yes & No \\
\hline Should people with HIV be allowed to get married? & 64 & 29 & 14 & 59 \\
\hline Should people with HIV be allowed to have children? & 48 & 43 & 10 & 66 \\
\hline $\begin{array}{l}\text { Should people with other inheritable conditions-eg, blindness, } \\
\text { deafness be allowed to have children? }\end{array}$ & 71 & 22 & 67 & 13 \\
\hline AIDS is a disease only of low class and illegal people & 23 & 73 & 45 & 40 \\
\hline $\begin{array}{l}\text { If you learnt that an acquaintance or colleague was HIV positive } \\
\text { would you be supportive? }\end{array}$ & 18 & 48 & 10 & 63 \\
\hline $\begin{array}{l}\text { If you learnt that an acquaintance or colleague was HIV positive } \\
\text { would you keep your distance? }\end{array}$ & 60 & 15 & 75 & 6 \\
\hline $\begin{array}{l}\text { Do you agree: I am willing to treat patients who are HIV positive } \\
\text { (including handling body fluids, operations, deliveries) }\end{array}$ & 62 & 30 & NA & NA \\
\hline $\begin{array}{l}\text { Do you agree: I would prefer not to treat patients who are HIV } \\
\text { positive }\end{array}$ & 81 & 10 & NA & NA \\
\hline
\end{tabular}


Table 4 Attitudes to HIV testing: responses by percentage

\begin{tabular}{|c|c|c|c|c|}
\hline & \multicolumn{2}{|c|}{ Health professionals } & \multicolumn{2}{|c|}{ Pregnant women } \\
\hline & Yes & No & Yes & No \\
\hline All pregnant women should have a free compulsory HIV test & 94 & 2 & 86 & 3 \\
\hline $\begin{array}{l}\text { All pregnant women should be offered a free test for HIV } \\
\text { with the right to refuse }\end{array}$ & 7 & 90 & 27 & 47 \\
\hline $\begin{array}{l}\text { A compulsory free HIV test should be added to the premarital } \\
\text { examination }\end{array}$ & 96 & 3 & 85 & 4 \\
\hline $\begin{array}{l}\text { A free HIV test with the right to refuse should be offered at the } \\
\text { premarital examination }\end{array}$ & 10 & 83 & 18 & 56 \\
\hline Would you agree to a free test for HIV? & 91 & 7.7 & 77 & 8 \\
\hline Would you want to know the result? & 98 & 1.3 & 78 & 8 \\
\hline If you were found to be HIV positive would you tell your partner? & 88 & 4 & 74 & 8 \\
\hline If you were found to be HIV positive would you tell your friends? & 12 & 70 & 7 & 65 \\
\hline $\begin{array}{l}\text { If you were found to have syphilis or other STI would you tell your } \\
\text { partner? }\end{array}$ & 80 & 4 & 66 & 10 \\
\hline $\begin{array}{l}\text { If you were found to have syphilis or other STI would you tell your } \\
\text { friends? }\end{array}$ & 5 & 90 & 4 & 75 \\
\hline $\begin{array}{l}\text { If you (or partner) tested positive for HIV would you want an } \\
\text { abortion? }\end{array}$ & 66 & 15 & 84 & 2.4 \\
\hline $\begin{array}{l}\text { If you had HIV and there was a free medicine to reduce the risk } \\
\text { of HIV in the baby would you accept it? }\end{array}$ & 71 & 17 & 86 & 2.6 \\
\hline
\end{tabular}

partner if they were positive (92\% and $76 \%$ respectively) and a lot less would tell anybody else (10\% and $7 \%)$. But these proportions were very similar to the numbers who would tell their partner if they tested positive for syphilis or other STIs. There were no significant differences in attitudes to testing between subgroups and there was no association with level of knowledge as defined by the knowledge scores.

Of particular note is the large proportion of professionals $(66 \%)$ and mothers (84\%) who regard termination as the best option if they themselves tested HIV positive in pregnancy, but almost as many would accept medication to reduce maternal to child transmission in preference to termination.

\section{DISCUSSION}

This study was carried out to determine the preparedness of these communities for the introduction of VCT programmes and specifically to determine what measures were necessary before implementation. The response rate was high. This is, firstly, because the Chinese are probably more used to complying with the demands of authority figures, secondly, because there is good awareness of HIV in these communities and pregnant women were told that the results of the study would contribute to improving HIV/AIDS services, and, thirdly, questionnaires were completed while women were waiting to be seen by doctors, so no time was sacrificed.

The study has a number of limitations. A more qualitative approach would have elucidated some of the reasons behind the bold statements. (This is an area we are currently exploring within the newly established VCT programmes.) Further the sample was predominantly female. Various logistical considerations prevented us from including a sample from premarital examination attendees, which would have redressed this balance. But a number of lessons have been learnt.

Firstly, levels of knowledge were good, especially given the low educational level of many of the pregnant women in the study. The levels we found were better than in previous reports from China. ${ }^{14-19}$ However, comparison is difficult because studies have been carried out in very different population groups. For example, whereas everybody in our study had heard of HIV, a study carried out in the year 2000 in rural Anhui province, where prevalence is low, showed that only $63 \%$ of respondents had even heard of HIV. ${ }^{20}$ We believe our respondents were better informed for two reasons: firstly, there is a general increase in awareness throughout China because of the recent more open approach adopted by the authorities and in the media and, secondly, our study was carried out in areas where the first cases of HIV were documented over 15 years ago and where there have been sporadic education campaigns over those years.

But there are still areas of weakness in the knowledge of health professionals, particularly in the area of maternal to child transmission and its prevention. This is perhaps more worrying given that they are all working in the maternal and child health field in high prevalence areas; and it clearly indicates that education for health professionals still needs to precede and accompany VCT programmes.

Secondly, the study shows that there is very considerable stigma associated with HIV. A high degree of stigma is something identified in other earlier studies ${ }^{10141517}$ and although our study results suggest there has been some improvement, this remains a barrier to testing and to treatment. In particular, stigma would pose serious problems for individuals identified positive in testing programmes. Attitudes are probably influenced by the fact that most of the known PLWHA in Yunnan Province are injecting drug users or (much less commonly) sex workers, both of which are officially illegal activities, although often tolerated. The intolerant attitudes towards marriage and child bearing in PLWHA need to be seen in the context of a society which has traditionally had the authority to prohibit marriage and child bearing. The latter power still exists, the former no longer since the change in the marriage law in October 2003 made the premarital examination voluntary. But the question which asks whether people with other inheritable conditions should be allowed to have children suggests a more general intolerance of divergence from societal norms and emphasises a cultural imperative to produce "normal" babies. ${ }^{21}$ Perhaps the attitudes towards HIV are simply an extension of this.

Health professionals, who perhaps should be taking a lead in the battle to destigmatise HIV/AIDS, are not much more enlightened. What these negative attitudes show is that education for health professionals and the general public must address these issues explicitly, and they need to be at the forefront of any educational programme. They further emphasise the importance of clear protocols. These must cover issues of confidentiality, specific guidelines for the management of non-concordant couples, clear guidance on the management of test positives, including the need to treat 


\section{Key messages}

- VCT is playing an increasing part in prevention and care strategies for HIV/AIDS in many countries and China has now recognised its importance. Essential prerequisites for successful VCT programmes include awareness and knowledge of HIV, acceptability of VCT to the community, and willingness of health professionals to treat HIV positive individuals

- In four high prevalence areas of Yunnan province, where VCT programmes were planned, attitudes towards HIV/AIDS and PLWHA were negative even among health professionals, $60 \%$ of whom would keep their distance from an HIV positive acquaintance, and $30 \%$ of whom would not be willing to treat an HIV positive patient

- There was strong support for compulsory HIV testing in pregnancy and at the premarital examination

- Community education programmes and intensive training of health workers must accompany VCT programmes and must particularly address negative attitudes towards HIV/AIDS and PLWHA

them with respect and without prejudice, and in pregnancy, clear guidance about advising women of the risks of maternal to child transmission and the choices available to reduce that risk. Our findings suggest this would reduce the number of women opting for termination.

Thirdly, testing is overwhelmingly supported by mothers and health professionals alike. The surprise is that compulsory testing is preferred over voluntary, and that testing at the premarital examination is more favoured. (This is somewhat ironic since the premarital examination is no longer compulsory.) Attitudes to testing are not related to knowledge levels in contrast to findings from elsewhere. ${ }^{22} \mathrm{We}$ believe that this unambiguous attitude towards testing needs to be seen in the context of a society where choice is limited in many aspects of life, and particularly in the area of reproduction. These findings indicate that the uptake of VCT could be expected to be very high.

Fourthly, there are three trends in the subgroups we studied. Younger health professionals, better educated women, and respondents in Derhong prefecture were consistently more enlightened. Clearly, the age effect in health professionals bodes well for the future. The failure to find such an effect in the pregnant women may be because of the relatively narrow age range covered, which is necessitated by the legal requirement for late marriage and small family size. The Derhong experience illustrates that sustained community education interventions can greatly influence both knowledge and attitudes even in a relatively uneducated population.

These lessons have been incorporated into two pilot VCT programmes which started in late 2002 in hospitals in Derhong and Kunming. They have been preceded by training for all health professionals and have been accompanied by community based education programmes which have particularly addressed negative attitudes towards HIV and PLWHA. Locally, appropriate protocols have been developed and a process of audit had been instigated which evaluates adherence to the agreed protocols.

\section{ACKNOWLEDGEMENTS}

This study was funded by the Wellcome Trust of the UK.

We thank the Maternal and Child Health Department of Yunnan Bureau of Public Health for supporting the implementation of this project and the research assistants for their conscientious and caring approach to the data collection among the pregnant women.

\section{CONTRIBUTORS}

All authors contributed to the design of the study; TH supervised the implementation of the study and wrote the paper; DL and LH implemented the study and carried out the data entry; AT corrected drafts of the paper.

\section{Authors' affiliations}

T Hesketh, A M Tomkins, Centre for International Child Health, Institute of Child Health, University College London, UK

L Duo, H Li, Yunnan Provincial Red Cross Hospital, Kunming, PR China

\section{REFERENCES}

1 UNAIDS. Present AIDS epidemic situation in China, 2002

(www.unchina.org/unaids).

2 US National Intelligence Council. The next wave of HIV/AIDS: Ethiopia, Nigeria, Russia, India and China, 2002. ICA 2002-04 D (www.odci.gov/nic).

3 Ministry of Public Health. The National HIV Sentinel Surveillance Group: national sentinel surveillance data for HIV infection in China for 2001, Beijing 2002.

4 Human Rights Watch. Locked doors: human rights of people living with HIV/ AIDS in China 2003;15(7)

5 Summers T, Spielberg F, Collins C, et al. Voluntary counselling, testing and referral for HIV: new technologies, research findings create dynamic opportunities. J Acquir Immune Defic Syndr 2000;25(Suppl 2):S128-35.

6 Pronyk PM, Kim JC, Makhubele MB, et al. Introduction of voluntary counselling and rapid testing for HIV in rural South Africa: from theory to practice. AIDS Care 2002; 14:859-65.

7 Dabis F, Newell ML, Fransen L, et al. Prevention of mother-to-child transmission of HIV in developing countries: recommendation for practice. Health Policy and Planning 2000;15:34-42.

8 De Cock KM, Johnson AM. From exceptionalism to normalisation: a reappraisal of attitudes and practice around HIV testing. BMJ 1998;316:290-3.

9 Hesketh T. Getting Married in China: pass the medical first. BMJ 2003;326:277-9.

10 Vermund SH, Wilson CM. Barriers to HIV testing-where next? Lancet 2002;360:1186-7.

11 Hesketh T, Huang XM, Wang ZB, et al. Using the premarital examination for population-based surveillance for HIV in China: a pilot study. AIDS 2003:88:467-71.

12 Wu ZY. Epidemics and Impact of AIDS and strategy for control in PR China, 2002. Presentation at Centre for Strategic and International Studies, Washington DC, 3 October 2002 (www.kaisernetwork.org/healthcast/csis/ oct2002)

13 Centre for Disease Control HIV/STD. Behavioural Surveillance Working group, 2000. Core measures for HIV/STD risk behaviour and prevention: questionnaire-based measurement for surveys (www.cdc.gov).

14 Anderson AF, Zheng Q, Wu G, et al. HIV knowledge and attitudes among health care professionals in Guangxi Zhuang Autonomous Region, China. Infect Control Hosp Epidemiol 2003;24:128-31.

15 Wu Z, Detels R, Ji G, et al. Diffusion of HIV/AIDS knowledge, positive attitudes and behaviours through training of health professionals in China. Aids Educ Prev 2002; 14:379-90.

16 Wu Z, Qi G, Zeng Y, et al. Knowledge of HIV/AIDS among health care professionals in China. AIDS Educ Prev 1999;11:353-63.

17 Zhang $X$, Luo $B$, Zhang K. A KABP study about AIDS among taxi-drivers and hotel attendants in Beijing. Chinese J Epidemiol 1994;1596:323-7.

18 Li VC, Cole BL, Zhang SZ, et al. HIV-related knowledge and attitudes among medical students in China. AIDS Care 1993;5:305-12.

19 Zhen XW, Zhang JP, Chen YL. A cohort study and KAP investigation of HIV infected persons in Ruili and other counties in Yunnan. Chinese J Epidemiol 1995; 16:67-70.

20 Liu H, Ma Z, Yu W. Attitude to voluntary HIV testing and result disclosure among rural residents in China. Zhonghua Yu Fang Yi Xue Za Zhi 2001;35:30-2.

21 Yuan R. Paying attention to eugenics and improving the quality of the population. Population Research 1996:36-8.

22 Kalichman SC, Simbayi LC. HIV testing attitudes, AIDS stigma and voluntary HIV counselling and testing in a black township in Cape Town, South Africa. Sex Transm Infect 2003;79:442-7. 